\title{
IDENTIFYING FACTORS ASSOCIATED WITH THE USE OF A SCALED-UP POSTPARTUM FAMILY PLANNING PROJECT IMPLEMENTED IN PUNJAB, PAKISTAN
}

\author{
Aniza Ismail ${ }^{1}$, Mariam Ashraf*2, Idayu Badilla Idris ${ }^{3}$, Inayat Thaver ${ }^{14}$, Ahsan Maqbool Ahmad ${ }^{15}$ and Sarmad \\ Jamal Siddique ${ }^{6}$ \\ ${ }_{1 \notin 3}$ Department of Community Health, Faculty of Medicine, Universiti Kebangsaan Malaysia Medical Centre (UKMMC), Jalan \\ Yaakob Latiff Bandar Tun Razak, 56000 Cheras, Kuala Lumpur, Malaysia \\ $2 \& 6$ PhD Scholars, Department of Community Health, Faculty of Medicine, Universiti Kebangsaan Malaysia Medical Centre \\ (UKMMC), Jalan Yaakob Latiff Bandar Tun Razak, 56000 Cheras, Kuala Lumpur, Malaysia \\ ${ }^{4}$ Department of Community Health Sciences, Bahria University Medical and Dental College, Defense Phase II, Karachi, \\ Pakistan \\ ${ }^{5}$ Health Services Academy, Chak Shehzad, Islamabad, Pakistan
}

${ }^{*}$ Corresponding author: Mariam Ashraf

Email: maryamashraf@hotmail.com

\begin{abstract}
Pakistan has one of the highest unmet needs for family planning among low and middle-income countries. Postpartum family planning (PPFP) is considered to have the potential of significantly improving the contraceptive prevalence rate and reducing the unmet need for family planning. Jhpiego (John Hopkins Affiliate) implemented a PPFP pilot project in the district headquarter hospital of Mandi Bahauddin. The key interventions of the Jhpiego pilot were to improve services pertinent to PPFP. Based on the successes of the pilot, the interventions were scaled up to other health facilities. The present study aims to identify the factors associated with the utilization of the postpartum family planning health project implemented in the health facilities once the scale-up activities ended and comparing it with the pilot facility. A crosssectional survey with a sample of 410 women using postpartum care services in the facilities was conducted using a structured questionnaire. The findings indicated that the complete set of interventions were not present in the facilities. Postpartum family planning method uptake was low (26\%) along with the shortage of contraceptives supplies. Multivariate logistic regression was conducted to identify the predictors for the current use of postpartum family planning. The predictors identified that women with the age of 25 years and above with 3 or more children, who attended antenatal care visits and were counselled on PPFP were more likely to adopt/use PPFP in the postpartum period. The findings indicated scale-up was limited and the interventions were not sustained once the donor support ended.
\end{abstract}

Keywords: Postpartum Family planning uptake, contraceptive use, project-based interventions

\section{INTRODUCTION}

Postpartum family planning is an intervention to fulfil unmet needs for family planning. Demographic and health survey data of 27 countries indicate that $65 \%$ of women who are $0-12$ months postpartum want to avoid pregnancy in the next 12 months but are not using contraception. Furthermore, research indicates that women who had delivered at a health facility had 1.4 times higher odds of contraceptive use than women who delivered at home. Those who received information about birth spacing from a doctor or relatives/friends had 1.81 and 1.38-times higher odds of contraceptive use respectively, as compared to those who did not ${ }^{1}$. Furthermore, women who have awareness of PPFP have four times higher odds $(\mathrm{OR}=3.7)$ to use PPFP compared with women who lack awareness. This positive association between knowledge and PPFP is well reported by other studies also $2,3,4$.
Pakistan has one of the highest unmet needs for family planning among low- and middle-income countries ${ }^{5}$. Though couples' desire for another child decreases with the birth of each child. An alarmingly low proportion of women (13\%) adopt a modern contraceptive method in the postpartum period. According to the Pakistan Demographic Health Survey (PDHS) 2017, the total fertility rate (TFR) in Pakistan is 3.6 births per woman. Women living in rural areas, on average, bear one more child than mothers in urban areas (3.9 versus 2.9 births per woman). TFR is highest among rural poorer women with the lowest levels of education ${ }^{6}$. To make the best use of public health research, interventions found to be effective in improving health need to be scaled up and delivered on a population-wide basis. The transfer of new knowledge from public health research into practice, however, continues to be sub-optimal. On average, it takes over 6 years for research evidence to reach reviews, papers, and textbooks, and a further 9 years for this evidence to be implemented 
into practice. Both the failure of effective public health initiatives to influence public health practice and the lag between evidence generation and implementation represent considerable impediments to population health improvement as it denies or delays community access to effective services ${ }^{7}$.

The strengthening and Sustaining Postpartum Family Planning project was implemented by an international non-governmental organization John Hopkins Affiliate (Jhpiego). The initial two-year (Sept 2011-Dec 2013) pilot project was carried out in the Mandi Bhauddin district. Jhpiego supported selected health facilities by equipping them with material resources, building service providers' capacity to provide PPFP services, introducing the concept of supportive supervision, and enhancing PPFP counselling during the antenatal and postnatal periods. In this pilot, the integrated approach improved the counselling and clinical skills of public sector health care providers resulting in increased acceptance of PPFP. The project noted a $23 \%$ increase in the uptake of interval intrauterine contraceptive devices (IUCDs) during the extended postpartum period. Based on the pilot results, it was later scaled up from January 2014 to October 2015 followed by an expansion from November 2015 to August 2017. The scale-up aimed to successfully demonstrate how the model could lead to a substantial increase in PPFP counselling services and family planning acceptance during the postpartum and post-abortion periods across the Punjab province.

The current study focuses on the utilization of PPFP services after the scale-up was completed and Jhpiego withdrew its support from the facilities. For this study Pilot study is defined as small study carried out in preparation for larger investigations and Scale-up refers to efforts to increase the impact of innovations successfully tested in pilot or experimental projects to benefit more people and to foster policy and program development on a lasting basis. This study includes the district headquarter hospital (DHQH) of Mandi Bahauddin in which the pilot was run for two years and the success of the interventions was proven and based on which the scale-up was done. The comparisons were made between the pilot and the scale-up districts which include DHQHs of Gujranwala, Dera Ghazi Khan (DG Khan) and Sargodha. The comparison was made to identify the difference of services in the pilot and scale-up health facilities and to identify the factors affecting the utilization of PPFP in the facilities.

\section{MATERIALS AND METHODS}

\section{Study sites \& design}

The study sites included the secondary level care hospitals which are the District Health Quarter Hospital (DHQH) of the four districts. This includes in which the pilot was conducted namely $\mathrm{DHQH}$ of Mandi Bahauddin and three scale-up districts namely Sargodha, DG Khan and Gujranwala. All these four districts belong to Punjab province and their socio-demographic characteristics are comparable. Furthermore, the geographical location of the health facilities belongs to periurban areas. The comparison was made between the pilot and the scale-up districts. A crosssectional exit survey in the form of structured interviews with the postpartum mothers utilizing the services of the facilities was carried out. The interviews were conducted at the time when women were leaving the facility after utilizing the services.

\section{Sampling population and sample size}

As the project interventions were specifically for postpartum clients the target population included Married Women of Reproductive Age group (MWRA) 18-49 years of age utilizing (postpartum care) services in the secondary care level facilities of the four districts. For the survey, a sample size of 381 was calculated using EPI info stat calc. The sample size was estimated based on $55 \%$ of women using the family planning method as per health survey ${ }^{6}$. This calculation was done considering a $5 \%$ significance level. Considering a non-response rate of $10 \%$ a total of 420 women were to be interviewed. The sample was equally divided in the 4 hospitals as the population coverage, the demographic characteristics and the project interventions were similar. A total sample of $\mathbf{4 4 0}$ was collected with 110 women interviewed from each hospital.

Systematic random sampling was done with a random start. An estimate of the number of women utilizing the postpartum care services was calculated using previous records (last 6 months). Based on that a number was generated using the following formula

Estimated number of clients to invite to respond per day $\equiv$

$[(\mathrm{n} / 100) *$ (\% expected refusal rate) $]{ }^{*} \mathrm{n}$

Number of days the facility operates per week (7) Approximately 5 women were interviewed on daily basis. The interviews were spread throughout the day.

\section{Inclusion and exclusion criteria for the research study}

All those married women of the reproductive age group (18-49 years) who gave consent and were utilizing the postpartum care services (immediately 
after delivery and up to 6 weeks ) were included in the survey. Women with spontaneous abortion were not part of the study.

\section{Ethical Approval}

Overall ethical approval of the study was obtained from the Research Ethics Committee, The National University of Malaysia (UKM PPI/111/8/JEP-2016596). Ethical approval was also sorted from the National Bioethics Committee, Pakistan Medical and Health Council, Islamabad, Pakistan reference no 4$87 /$ NBC-262/17/4376. A letter was also issued from Jhpiego for approval to study the project.

Informed consent was taken by all the participants of this research

\section{Study instrument}

For the cross-sectional survey, the women's Postpartum Client interview questionnaire for Family planning postpartum client interview questionnaire was derived from Jhpiego validated questionnaire $^{1}$ for women receiving postpartum care. The tool was already used for collecting information in Pakistani settings. The tool used for the clients was translated in Urdu as English is the secondary language. English language versions of the tool were translated into the local language of Urdu, by an independent person who was not directly involved in this research project. The final versions of these forms were then back-translated into English by a separate independent person. The back-translated versions were compared and reviewed for consistency with the original English version of these forms. Any inconsistencies found through this comparison were rectified between the back-translated and Urdu versions of the data collection tools., the health care providers and the project team members were well versed with English. It consisted of a total of sixty-three questions with sub-questions.

The information collected included sociodemographic characteristics, women's age, education status and years of formal education (for those who had attended school), duration of the marriage, occupation. This also included information about the spouse. Additional information related to the household was also acquired including average monthly household income, number of family members, number of earning members, area of residence, availability of various assets in the household $(\mathrm{HH})$. Furthermore, information pertinent to use of antenatal care services, history related to existing and desire for children, use of family planning methods, intention to adopt any method, overall satisfaction with the services and reasons to prefer the facility was collected.

\section{DATA COLLECTION AND ANALYSIS}

Data collection took place from January 2018 to April 2018 in all four districts. Data were collected through face-to-face interviews in the field on paper-based forms. Systematic random sampling was done with a random start. The raw data which was collected from the respondents were entered to excel and then necessary checks were put in to ensure minimal data entry errors. Once entered, data cleaning was carried out. All the quantitative data was then transferred to SPSS version 19 for further analysis. Means and standard deviations for continuous variables and frequencies for categorical variables were calculated. P-value was calculated using chi-square.

\section{RESULTS}

\section{Socio-demographic characteristics}

The data indicate that a significantly higher proportion of younger age category women, living in the nuclear family system and belonging to higher income groups from the scale-up district were using the facility. A significant comparison was consistent across the stratum of monthly income, as well as when compared across the wealth quintiles. When compared for the strata of the number of children and desire for more children, a higher proportion of women with fewer children in the scale-up district as compared to the pilot district were current users of the family planning method. It may hence be derived, that in comparison to the pilot district, a higher proportion of younger, less affluent, those living in a nuclear family system, and having a lesser number of children was currently utilizing the facility in scale-up districts. (Table 1).

\section{Reasons for preferring this facility}

When comparing the reason for using the facility, affordability was the main reason as reported by the women from the pilot district to prefer to choose services from this facility. As we can see that participants were from a low socio-economic background from the pilot district so affordability was the utmost important factor for women to choose this facility. A mix of factors was identified by the respondents from the scale-up districts. (Table 2). 
Table 1: Comparison of sociodemographic characteristics between Pilot and scale-up districts

\begin{tabular}{|c|c|c|c|c|c|c|c|}
\hline \multirow[t]{2}{*}{ Sociodemographic } & \multirow{2}{*}{$\begin{array}{c}\text { Frequency } \\
\mathrm{N}\end{array}$} & \multicolumn{2}{|c|}{ Pilot } & \multicolumn{2}{|c|}{ Scale up } & \multirow[t]{2}{*}{$x^{2}$} & \multirow[t]{2}{*}{ P-value } \\
\hline & & No & $\%$ & No & $\%$ & & \\
\hline \multicolumn{8}{|l|}{ Age in years } \\
\hline $18-25$ & 149 & 30 & $(27)$ & 119 & (36) & 2.84 & 0.09 \\
\hline$>25$ & 291 & 80 & (73) & 211 & (64) & & \\
\hline \multicolumn{8}{|l|}{ Marriage duration in years } \\
\hline $1-5$ & 187 & 33 & (30) & 154 & $(47)$ & 16.9 & $0.00^{*}$ \\
\hline $6-10$ & 133 & 31 & (28) & 102 & (31) & & \\
\hline$>10$ & 120 & 46 & $(42)$ & 74 & (22) & & \\
\hline \multicolumn{8}{|l|}{ Education status of women } \\
\hline No education & 120 & 35 & (32) & 85 & $(26)$ & 13.3 & $0.01^{*}$ \\
\hline Primary & 170 & 52 & (47) & 118 & (36) & & \\
\hline Middle & 96 & 18 & (16) & 78 & (24) & & \\
\hline Secondary & 35 & 4 & (4) & 31 & (9) & & \\
\hline Higher & 19 & 1 & (1) & 18 & (5) & & \\
\hline \multicolumn{8}{|l|}{ Husband education } \\
\hline No education & 70 & 26 & (24) & 44 & (13) & 25.9 & $0.00^{*}$ \\
\hline Primary & 98 & 34 & (31) & 64 & (20) & & \\
\hline Middle & 110 & 31 & (28) & 79 & (24) & & \\
\hline Secondary & 71 & 10 & (9) & 61 & (19) & & \\
\hline Higher & 89 & 9 & (8) & 80 & (24) & & \\
\hline \multicolumn{8}{|l|}{ Woman occupation } \\
\hline Housewife & 405 & 107 & $(97)$ & 298 & $(90)$ & 5.47 & $0.01^{*}$ \\
\hline Working & 35 & 3 & (3) & 32 & (10) & & \\
\hline \multicolumn{8}{|l|}{ Husband occupation } \\
\hline Farming & 76 & 8 & (7) & 68 & $(21)$ & 26.7 & $0.00^{*}$ \\
\hline Laborer & 201 & 73 & $(66)$ & 128 & (39) & & \\
\hline Private service & 117 & 21 & (19) & 96 & (29) & & \\
\hline Govt service & 46 & 8 & $(7)$ & 38 & (12) & & \\
\hline \multicolumn{8}{|l|}{ Family type } \\
\hline Nuclear & 234 & 51 & $(46)$ & 183 & (55) & 7.36 & $0.00^{*}$ \\
\hline Joint & 206 & 59 & (54) & 147 & (45) & & \\
\hline \multicolumn{8}{|l|}{ Locality } \\
\hline Rural & 222 & 36 & (33) & 186 & (56) & 23.5 & $0.00^{*}$ \\
\hline Urban & 218 & 74 & $(67)$ & 144 & (44) & & \\
\hline \multicolumn{8}{|l|}{ Monthly income in PKR } \\
\hline Up to 20,000 & 270 & 97 & $(88)$ & 173 & $(52)$ & 44.4 & $0.00^{*}$ \\
\hline$>20,000$ & 170 & 13 & $(12)$ & 157 & $(48)$ & & \\
\hline \multicolumn{8}{|l|}{ Wealth Index } \\
\hline Lowest & 34 & 15 & $(14)$ & 19 & $(6)$ & 27.4 & $0.00^{*}$ \\
\hline Second & 129 & 40 & (36) & 89 & (27) & & \\
\hline Middle & 182 & 49 & (45) & 133 & $(40)$ & & \\
\hline Fourth & 82 & 6 & (5) & 76 & (23) & & \\
\hline Highest & 13 & 0 & (0) & 13 & $(4)$ & & \\
\hline \multicolumn{8}{|l|}{ No of children } \\
\hline $1-2$ & 217 & 39 & $(35)$ & 178 & (54) & 11.2 & $0.00^{*}$ \\
\hline$>3$ & 223 & 71 & (65) & 152 & (46) & & \\
\hline \multicolumn{8}{|l|}{ Desire for children } \\
\hline $1-2$ & 42 & 2 & $(2)$ & 40 & $(12)$ & 42.0 & $0.00^{*}$ \\
\hline $3-5$ & 248 & 44 & $(40)$ & 204 & $(62)$ & & \\
\hline More than 5 & 18 & 6 & (5) & 12 & $(4)$ & & \\
\hline up to God & 132 & 58 & (53) & 74 & (22) & & \\
\hline
\end{tabular}


Table 2: Reasons for using the facility

\begin{tabular}{|c|c|c|c|c|c|c|c|}
\hline \multirow[t]{2}{*}{ Variables } & \multirow{2}{*}{$\frac{\text { Frequency }}{\mathrm{N}}$} & \multicolumn{2}{|c|}{ Pilot } & \multicolumn{2}{|c|}{ Scale-up } & \multirow[b]{2}{*}{$\chi^{2}$} & \multirow[b]{2}{*}{ P-value } \\
\hline & & No & $\%$ & No & $\%$ & & \\
\hline \multicolumn{8}{|l|}{ Services are affordable } \\
\hline No & 171 & 26 & (24) & 145 & (44) & 14.3 & $0.00^{*}$ \\
\hline Yes & 269 & 84 & (76) & 185 & (56) & & \\
\hline \multicolumn{8}{|l|}{ Services are easily accessible } \\
\hline No & 256 & 59 & (54) & 197 & $(60)$ & 1.24 & 0.26 \\
\hline Yes & 184 & 51 & (46) & 133 & (40) & & \\
\hline \multicolumn{8}{|l|}{ Services are of high quality } \\
\hline No & 297 & 93 & $(85)$ & 204 & $(62)$ & 19.4 & $0.00^{*}$ \\
\hline Yes & 143 & 17 & (15) & 126 & (38) & & \\
\hline \multicolumn{8}{|l|}{ Provider skills are very good } \\
\hline No & 307 & 93 & $(85)$ & 214 & $(65)$ & 15.1 & $0.00^{*}$ \\
\hline Yes & 133 & 17 & (15) & 116 & (35) & & \\
\hline
\end{tabular}

${ }^{*} p$-value significant $<0.05$

Postpartum family planning information

Most of the women in the pilot district received information from their health care provider about postpartum family planning. A similar but lesser woman reported from scale-up districts receiving such information. When asked specifically about
PPIUCD a lesser percentage in scale-up districts was aware of it and that it can be immediately used after birth. However, the preference of choosing this method was low across all the districts but this finding was not significant. (Table 3)

Table 3: Information provision on PPFP

\begin{tabular}{|c|c|c|c|c|c|c|c|c|}
\hline \multirow{2}{*}{$\begin{array}{l}\text { Variable } \\
\text { PPFP information }\end{array}$} & & \multirow{2}{*}{$\frac{\text { Frequency }}{\mathrm{N}}$} & \multicolumn{2}{|c|}{ Pilot } & \multicolumn{2}{|c|}{ Scale-up } & \multirow[b]{2}{*}{$\chi^{2}$} & \multirow[b]{2}{*}{ P-value } \\
\hline & & & No & $\%$ & No & $\%$ & & \\
\hline \multicolumn{9}{|c|}{ Discussion on PPFP with HCP } \\
\hline & No & 208 & 45 & $(41)$ & 163 & (54) & 5.50 & $0.01^{*}$ \\
\hline & Yes & 204 & 65 & (59) & 139 & (46) & & \\
\hline \multicolumn{9}{|c|}{ Awareness of PPIUCD } \\
\hline & No & 246 & 52 & $(49)$ & 194 & $(60)$ & 4.45 & $0.03^{*}$ \\
\hline & Yes & 183 & 55 & (51) & 128 & (40) & & \\
\hline \multicolumn{9}{|c|}{$\begin{array}{l}\text { Preference of using PP IUCD over } \\
\text { other methods }\end{array}$} \\
\hline & No & 78 & 4 & $(67)$ & 74 & (77) & 0.34 & 0.56 \\
\hline & Yes & 24 & 2 & (33) & 22 & (23) & & \\
\hline
\end{tabular}

${ }^{*} p$-value significant $<0.05$

\section{Current user and intention to use}

In the pilot district, a significantly greater number of women were found to be using the PPFP method when compared to scale-up districts. One possible reason is that women in pilot districts were found to be those who had a greater number of children and were from the older age group compared to the scale-up districts. Among the methods use, tubal ligation was reported by a larger percentage of women in the pilot district compared to scale-up districts. Besides, the subdermal implant was found to be available only in the pilot district and was not available in the scale-up district. A higher percentage of the women had the intention to use a contraceptive method within one month time. (Table 4).

\section{Decision Making}

In all the districts husband was found to be the key influence in taking up the decision for adopting a method and to some extent parents in law as reported by the women. (Table 5) 
Table 4: current user and intention to use PPFP

\begin{tabular}{|c|c|c|c|c|c|c|c|}
\hline & \multirow{2}{*}{$\begin{array}{c}\text { Frequency } \\
\mathrm{N}\end{array}$} & \multicolumn{2}{|c|}{ Pilot } & \multicolumn{2}{|c|}{ Scale-up } & \multirow[t]{2}{*}{$x^{2}$} & \multirow[t]{2}{*}{ P-value } \\
\hline & & No & $\%$ & No & $\%$ & & \\
\hline \multicolumn{8}{|l|}{ Current user } \\
\hline No & 326 & 61 & (55) & 265 & $(80)$ & 26.5 & $0.00^{*}$ \\
\hline Yes & 114 & 49 & (45) & 65 & (20) & & \\
\hline \multicolumn{8}{|l|}{ Method type } \\
\hline subdermal implant & 6 & 6 & $(12)$ & 0 & $(0)$ & 9.29 & $0.01^{*}$ \\
\hline PPIUCD & 83 & 31 & (63) & 52 & (80) & & \\
\hline Tubal Ligation & 25 & 12 & (24) & 13 & (20) & & \\
\hline \multicolumn{8}{|l|}{ Intention to use FP } \\
\hline No & 64 & 14 & $(25)$ & 50 & $(24)$ & 0.02 & 0.86 \\
\hline Yes & 201 & 42 & (75) & 159 & (76) & & \\
\hline \multicolumn{8}{|l|}{ Time to use } \\
\hline within 1 month & 45 & 0 & $(0)$ & 45 & (36) & 20.0 & $0.00^{*}$ \\
\hline$>1$ month & 119 & 40 & $(100)$ & 79 & (64) & & \\
\hline
\end{tabular}

Table 5: Influence in decision making

\begin{tabular}{|c|c|c|c|c|c|c|c|}
\hline & & \multicolumn{2}{|c|}{ Pilot } & \multicolumn{2}{|c|}{ Scale-up } & \multirow{3}{*}{$\chi^{2}$} & \multirow{3}{*}{ P-value } \\
\hline Variables & Frequency & & & & & & \\
\hline & $\mathbf{N}$ & No & $\%$ & No & $\%$ & & \\
\hline \multicolumn{8}{|l|}{ Parents' in law } \\
\hline 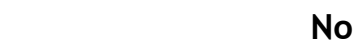 & 321 & 97 & $(92)$ & 224 & $(70)$ & 20.1 & $0.00^{*}$ \\
\hline Yes & 106 & 9 & (8) & 97 & (30) & & \\
\hline \multicolumn{8}{|l|}{ Husband } \\
\hline No & 7 & 3 & (3) & 4 & (1) & 1.24 & 0.26 \\
\hline Yes & 420 & 103 & (97) & 317 & (99) & & \\
\hline \multicolumn{8}{|l|}{ Doctors / Nurses } \\
\hline No & 403 & 94 & (89) & 309 & (96) & 8.63 & $0.00^{*}$ \\
\hline Yes & 24 & 12 & (11) & 12 & (4) & & \\
\hline
\end{tabular}

${ }^{*}$-value significant $<0.05$

LOGISTIC REGRESSION MODEL FOR POSTPARTUM FAMILY PLANNING

Multivariate logistic regression analysis was conducted to identify the predictors for the current use of postpartum family planning among the participants with the approach of parsimonious model building and screening criteria of $p$-value
$<0.20$ at the univariate level and $<0.05$ at the multivariate level. The model preparation was subjected to the Hosmer and Lemeshow goodness of fit test. The final derived multivariate model was found to be a good fit ( $p$-value $<0.80$ ) indicating a good fitness of model. (Table 6 )

Table 6: Logistic regression model for postpartum family planning women

\begin{tabular}{lcccc}
\hline Characteristic & B & OR - Adj & $\mathbf{9 5 \% ~ C l ~ o f ~ O R ~}$ & P-value \\
\hline Age $>$ 25 years & 1.18 & 3.26 & $1.32-8.04$ & 0.01 \\
No of children $>3$ & 1.85 & 6.41 & $3.12-13.1$ & 0.00 \\
No ANC visits in last pregnancy & -2.16 & 0.11 & $0.02-0.66$ & 0.01 \\
Received information on PPFP by HCP & 1.19 & 3.30 & $1.77-6.15$ & 0.00 \\
Awareness about PPIUCD & 2.37 & 10.7 & $5.57-20.9$ & 0.00 \\
Past user & 1.53 & 4.65 & $1.5-14.3$ & 0.00 \\
\end{tabular}

${ }^{*}$ Hosmer and Lemeshow Goodness of Fit test $p$-value $<0.80$

It was found that women of more than 25 years of age were 3.2 times more likely to adopt a PPFP method than the women in the younger age group. Similarly, women having more than 3 children were
6.4 times more likely to use the method than those having 2 or a smaller number of children. In terms of knowledge and awareness about PPFP, it was found that women who did not have any ANC visits 
were 2.16 times less likely to adopt any method in the postpartum period than the woman who had ANC visits. In addition, those who were counselled and received information on PPFP by the health care provider were 3.3 times more likely to adopt the method. Similarly, those who were aware of PPIUCD were more likely to adopt the method than those who were not aware of PPIUCD that it can be immediately used after delivery or within the postpartum period and past contraceptive users are more likely to choose a method. From the above it may hence be derived, that women with an age of 25 years and above having 3 or more children, who had attended ANC visits and were counselled on PPFP and were aware of the use of PPIUCD are more likely to adopt/use PPFP in the postpartum period.

\section{DISCUSSION}

The findings from the current study indicated that a complete set of interventions as per plan in the pilot phase of the project were not present in the scale-up districts. A subset of interventions that were present included trained staff, relevant equipment availability of information and education materials but none of the facilities had a complete set of contraceptive supplies. The facility was accessible as reported by $42 \%$ of the women yet the number of women who were adopting the postpartum family planning methods was less in all the districts. Overall postpartum method uptake was found to be only $26 \%, 114$ users out of a sample size of 440. If we look at individual findings from the districts the contraceptive uptake was better in Mandi Bahauddin, the pilot district and was found to be $45 \%$ compared to scale-up districts which were found to be $16 \%, 20 \%$ and $23 \%$ in Sargodha, Gujranwala and DG. Khan respectively. These findings are similar to the overall uptake of family planning methods in Pakistan which is $25 \%$ for any of the modern methods ${ }^{6}$.

Our study findings indicate that women of more than 25 years of age were 3.2 times more likely to adopt a PPFP method than the women in the younger age group. Similarly, women having more than 3 children were 6.4 times more likely to use the method than those having 2 or a smaller number of children. This finding is in relation to one of the studies conducted in Mexico which showed that parity positively affected postpartum contraceptive intake, 68\% PPIUCD and 29\% sterilization ${ }^{8}$. The current study also indicated tubal ligation as one of the popular methods mainly adopted by women having children 5 or more.

The current study findings indicate that women who did not have any ANC visits were 2.16 times less likely to adopt any method in the postpartum period than the woman who had ANC visits. In addition, those who were counselled and received information on PPFP by the health care provider were 3.3 times more likely to adopt the method. Similarly, those who were aware of PPIUCD were 4.65 times more likely to adopt the method than those who were not aware of PPIUCD that it can be immediately used after delivery or within the postpartum period. Our findings further indicate that majority of $204(46 \%)$ of women reported that they did not have any discussion/counselling on postpartum family planning, however, on the other hand, most of the women reported attending ANC sessions. Therefore, our study indicates that attending ANC sessions does not mean that one will be counselled on postpartum family planning. Not all women reported discussing PPFP. Literature indicates that antenatal counselling or intensity of antenatal care is associated with contraceptive use $^{9}$. Similarly, another study carried out in Kenya and Zambia strongly recommend that the promotion of ANC services should be considered as a mechanism to promote postpartum FP use and showed significant association with ANC service use and uptake of postpartum family planning use ${ }^{11}$. Studies further indicate that counselling for family planning during the antenatal period, is a standard of care but sadly is offered to a small portion of women in developing countries, where only a few receive effective antenatal care ${ }^{10}$.

Considering the findings in the current study a lot of gaps were identified indicating that a strong political will at the government level is required to ensure an adequate and sustained supply of contraceptives. Literature indicates that lessons learnt from conducting a pilot project and for those responsible for its scale-up are that they test and implement only those interventions that they can afford to scale up. There is no point in testing interventions that are not affordable. When designing a pilot project, planners should attempt to estimate the costs of scaling up before embarking on the planning phase. In so doing, the waste associated with piloting unsustainable interventions can be avoided. It may be that affordable interventions produce less remarkable results during the pilot phase, but being able to scale up more modest, affordable interventions will make a larger health impact than will small pilots that yield large health benefits for a short time only ${ }^{11}$. Literature indicates that once the pilot is scaled, the goal is to sustain the intervention so that benefits proven at a small scale can yield measurable health impact ${ }^{12}$.

\section{LIMITATIONS}

Primarily, the scale-up of the pilot project was studied retrospectively and the data collection took place four months after the scale-up activities were completed. Secondly, for assessing the quality of 
services through the cross-sectional survey, the research did not cover in-depth information about the contraceptive counselling received by the postpartum clients during their visits or at any time while meeting the health care provider from the antenatal period up to the postpartum period. Furthermore, no such observations were made pertinent to content and depth of counselling, time spent with the provider, where counselling occurred (e.g., delivery hospitalization, postpartum outpatient care visit), or the number of times women were counselled. Thirdly, as the data was self-reported by women up to 6 weeks after delivery, responses might be subject to social desirability or recall bias. Additionally, the survey did not determine the use of the lactation amenorrhea method, as the main focus was on the adoption of the contraceptive method during the postpartum period. Lastly, the project was scaled up to more districts of Punjab but the research was able to report the findings from only three scale-up districts, which limits the generalizability of the findings.

\section{CONCLUSION}

The current research findings indicated that the overall uptake of PPFP was low in all the districts mainly owing to lack of counselling, lack of supplies and lack of awareness of PPIUCD methods. The findings indicate a need for more clear and comprehensive planning for scaling-up pilot projects especially in Pakistan where resource constraints overall affect the sustainability of any project.

\section{ACKNOWLEDGEMENTS}

The author acknowledges the support provided by the research ethics committee of UKM and Pakistan to conduct the research. The author would also like to thank the then Country Director of Jhpiego Pakistan who permitted to conduct the research.

Competing interests: None to declare

Funding: None to declare

\section{REFERENCES}

1. Tappis H, Kazi A, Hameed W, et al. The role of quality health services and discussion about birth spacing in postpartum contraceptive use in Sindh, Pakistan: A multilevel analysis. PLOS One. 2015;10(10):1-18.

2. Palamuleni ME. Socio-economic and demographic factors affecting contraceptive use in Malawi. Afr J Reprod Health. 2013;17(3):91-104.
3. Thapa K, Dhital R, Rajbhandari S, et al. Improving post-partum family planning services provided by female community health volunteers in Nepal: A mixedmethods study. BMC Health Serv Res. 2020;20(1):1-13.

4. Wulifan JK, Brenner S, Jahn A, et al. A scoping review on determinants of unmet need for family planning among women of reproductive age in low and middle-income countries. BMC Women's Health [Internet]. 2016;16(1). Available from: http://dx.doi.org/10.1186/s12905-015$0281-3$

5. Moore Z, Pfitzer A, Gubin R, et al. Missed opportunities for family planning: An analysis of pregnancy risk and contraceptive method use among postpartum women in 21 low- and middle-income countries. Contraception [Internet]. 2015;92(1):31-9. Available from: http://dx.doi.org/10.1016/j.contraception .2015.03.007

6. NIPS. Pakistan Demographic Health Survey [Internet]. 2017. Available from: http://nips.org.pk/abstract_files/PDHS 2017-18 - key findings.pdf

7. Milat AJ, Newson R, King L. Increasing the scale of population health interventions: A Guide. Evid Eval Guide Ser Popul Public Heal Div. 2014;

8. Darney BG, Sosa-Rubi SG, Servan-Mori E, Rodriguez MI, Walker D, Lozano R. The relationship of age and place of delivery with postpartum contraception prior to discharge in Mexico: A retrospective cohort study. Contraception [Internet]. 2016;93(6):478-84. Available from: http://dx.doi.org/10.1016/j.contraception .2016.01.015

9. Do M, Hotchkiss D. Relationships between antenatal and postnatal care and postpartum modern contraceptive use: Evidence from population surveys in Kenya and Zambia. BMC Health Serv Res. 2013;13(1).

10. Villar J, Ba'aqeel H, Piaggio G, et al. WHO antenatal care randomised trial for the evaluation of a new model of routine antenatal care. Lancet (London, England) [Internet]. 2001 May 19 [cited 2019 Oct 18];357(9268):1551-64. Available from: 
http: / /www.ncbi.nlm.nih.gov/pubmed/11

377642

11. Janowitz B, Bratt J, Homan R, et al. How much will it cost to scale up a reproductive health pilot project? Reprod Health. 2007;(8).

12. Simmons R, Fajans $P$, Ghiron L. Scaling up Health Service Delivery - From pilot innovations to policies and programmes. Public Health [Internet]. 2007;123(9):6389. Available from: http: / / linkinghub.elsevier.com/retrieve/pi i/S0033350609001541 